\title{
Development of Amplitude-Controlled Parallel-Fatigue-Test System for Micro-Electromechanical Resonators
}

\author{
Tsuyoshi Ikehara* and Toshiyuki Tsuchiya $^{1}$ \\ National Institute of Advanced Industrial Science and Technology (AIST), \\ 1-2 Namiki, Tsukuba, Ibaraki 305-8564, Japan \\ 'Department of Micro Engineering, Kyoto University, \\ Yoshida Honmachi, Sakyo-ku, Kyoto 606-8501, Japan
}

(Received April 2, 2009; accepted August 13, 2009)

Key words: fatigue test, silicon, MEMS, resonator, amplitude control, parallel test

Fatigue in silicon has become an important issue for ensuring the reliability of micromechanical systems. However, the fatigue characteristics of micrometer-scale silicon specimens have not yet been clearly understood because of the difficulties in fatigue testing. Here, the authors describe the design, fabrication, measurement, and tuning of a fatigue test system developed for deflection-amplitude-controlled parallel testing using self-oscillation. The amplitude-controlled oscillation was fundamental for performing the fatigue test reliably and the tuning of the time constant was crucial to obtaining stable oscillation considering the mechanical response of the test device. As the electric system was integrated on a small circuit board and had a simple interface with a control computer, it was easy to fabricate a scaled-up, parallel-test system. Up to fourfold parallel testing was actually performed. A problem with the parallel testing system concerning signal interference was reported. Lifetime measurement and the acquisition of a deflection signal at fracture were successfully performed using the test system.

\section{Introduction}

Micro-electromechanical systems (MEMS) technologies have been used to fabricate various kinds of microsensor such as pressure sensors and accelerometers. Silicon is one of the most commonly used materials for MEMS structures, in both single-crystal and polycrystalline forms. Currently, we can use silicon wafers with a very high purity and an almost perfect crystal quality, and have considered its material properties to be well known. However, silicon still has unknown mechanical properties, such as fatigue on the nanometer to micrometer scale. Bulk silicon has been considered to exhibit practically no fatigue (subcritical crack growth) at room temperature because of its negligible plasticity and chemical stability. ${ }^{(1,2)}$ However, after the report on the frequency change

${ }^{*}$ Corresponding author: e-mail: ikehara.t@aist.go.jp 
measurement in a microresonator by Connally and Brown, ${ }^{(3)}$ the fatigue characteristics of silicon have attracted the attention of material researchers, and simultaneously posed difficulties for MEMS device designers. Various fatigue tests have been reported for silicon microspecimens, e.g., tests using devices including actuators, ${ }^{(4-6)}$ tests using external actuators, ${ }^{(7,8)}$ and tests using conventional test machines modified for microspecimens. ${ }^{(9-12)}$ Thus far, almost all fatigue tests on microscale silicon have shown the presence of fatigue in silicon in air even at room temperature. These reports have also shown difficulties in the precise deflection/stress measurement of a small test specimen, in the accurate amplitude control of the loading, and in test specimen preparation. The measured lifetimes usually have a large scattering that makes the analysis and comparison of measurement results difficult. The mechanism of fatigue in silicon is still uncertain although the effects of humidity in the environment on the fatigue life have been well elucidated..$^{7,13,14)}$ Various measurements have been performed to investigate the origin of fatigue in silicon. ${ }^{(15-18)}$

We have proposed the use of the self-oscillation of microresonators for lifetime measurement. One of the authors (T. T.) measured the fatigue lifetime of an accelerometer-like device using an external actuator and confirmed the effects of the environment. (7) We developed a fan-shaped fatigue test device of the type first proposed by Muhlstein et al. $^{(5)}$ and also an all-electric fatigue test system based on amplitudecontrolled self-oscillation. Thus far, such resonator devices have usually been operated by an external ac source adjusted at the natural frequency without feedback control. A complicated adjustment procedure was required when the natural frequency of the resonator changed during the fatigue test. ${ }^{(13)}$ Such frequency shift can cause the change in the vibration amplitude. However, our new system needs no frequency adjustment because the self-oscillation frequency automatically follows the natural frequency even if it is shifted by the occurence of subcritical crack growth or plastic deformation in the specimen. Therefore, the self-oscillation method along with the amplitude control function are expected to minimize the uncertainty in the amplitude measurement/control of microscale testing. On the other hand, it is difficult for such test methods to show frequency-dependent fatigue properties since the operation frequency is fixed at the natural frequency of the test device.

As a result of the amplitude-controlled self-oscillation method, we can easily fabricate a parallel-test system for efficient lifetime measurement because each test system becomes a compact, adjustment-free, and all-electric circuit. Lifetime measurement results using such a test system have already been provided in previous reports. ${ }^{(14,19)}$ However, we have not described the test system in detail. We report the design, fabrication, measurement, tuning procedures, and parallel operation of this fatigue test system in this paper.

\section{Design of the Test System}

\subsection{Test device}

Our test system employs the self-oscillation of a microresonant device at its resonance frequency. Any type of resonator device can be used provided self-oscillation 
is available with a feedback electric circuit. Generally, microresonators have an actuator to provide vibration and a sensor of output deflection inside the devices. By satisfying the conditions of feedback oscillation, a loop gain equal to 1 and phase matching, the resonator can start self-oscillation at its resonance frequency. The actuator and sensor are not necessarily inside the device. For example, devices or instruments outside the device can be employed, such as a piezoelectric vibrator as an actuator or a laser displacement meter as a sensor. The composition of a self-oscillation circuit is strongly dependent on the devices, actuators, and sensors used.

We show an example of a fatigue test device having two comb electrodes as an electrostatic actuator and a sensor in this paper. A micrograph of the device made of single-crystal silicon of $5 \mu \mathrm{m}$ thickness is shown in Fig. 1. As the fan-shaped resonator of $250 \mu \mathrm{m}$ radius vibrates at a natural frequency of about $39 \mathrm{kHz}$ (quality factor $Q=$ 370 in air), the test piece at the pivot is subjected to high-cycle repetitive bending. The delayed fracture of the test piece has been studied as the fatigue of single-crystal silicon. The structure and characteristics of the device have been described in detail in previous reports. ${ }^{(14,19)}$ The effect of gravity can be neglected because the force applied to the resonator is estimated to be less than $1 \%$ of the electrostatic actuation force.

A block diagram of the test system for a single specimen is shown in Fig. 2. The test device has an electrostatic sensor that outputs a charge proportional to the deflection angle. The output was detected by a typical charge amplifier and processed with amplification and phase control to satisfy the oscillation conditions. As the phase of the sensor-output signal was retarded by $\pi / 2$ from the actuation signal at resonance, we inserted a variable phase shifter in the feedback circuit and made appropriate adjustments. The loop gain was also adjusted, as described in the next subsection. The amplitude of the processed signal was controlled using an automatic gain controller (AGC) and fed to an amplifier to drive the electrostatic actuator. As the electrostatic actuator required high driving voltages, we used a high-voltage operational amplifier (OPA445; Burr-Brown) with a power supply of $\pm 40 \mathrm{~V}$ with biasing.

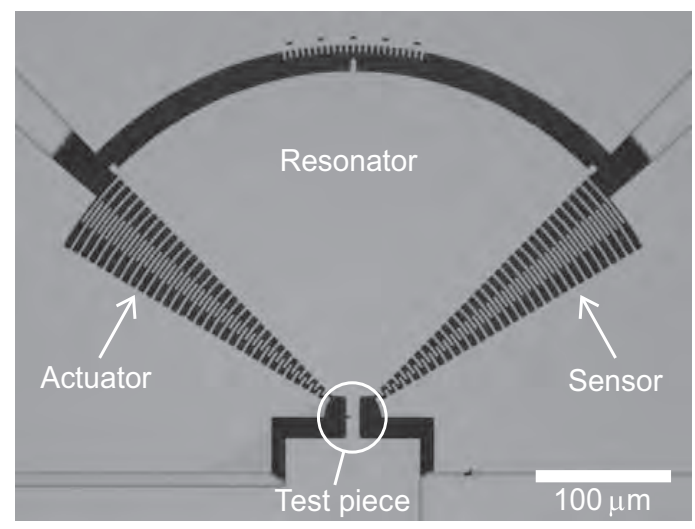

Fig. 1. Micrograph of fatigue test device. 


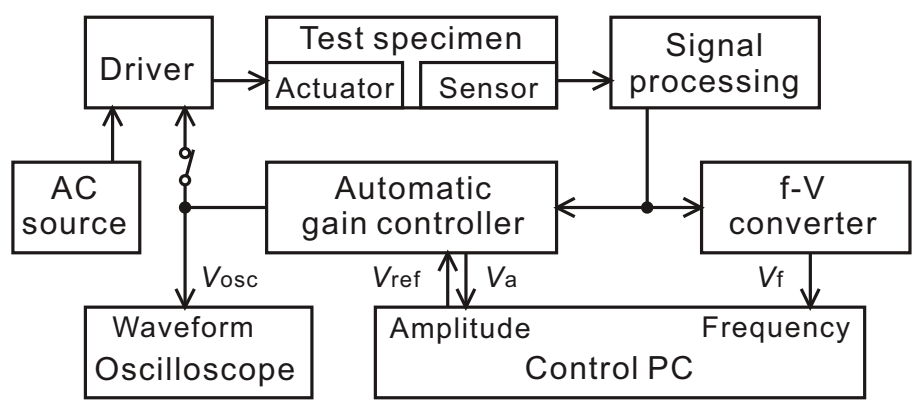

Fig. 2. Block diagram of fatigue test system for single specimen.

The test system stabilizes the vibration amplitude during the test using the AGC function controlled by a computer. The computer also acquires the vibration conditions of the test device at regular intervals. In our system, the amplitude and frequency of the oscillation are measured and recorded. Additionally, the waveform $V_{\text {osc }}$, which is proportional to the deflection angle of the resonator, is observed using an oscilloscope. The absolute value of the deflection angle was calibrated by observing an image of moving resonators directly under a microscope.

\subsection{Amplitude control}

The fundamental feature of this system is the AGC because the fatigue test is best conducted under a constant-loading condition. The vibration amplitudes of the resonators easily diverge in the self-oscillation state enhanced by the quality factor, and are difficult to maintain at a constant level with no amplitude control. Therefore, we inserted the AGC circuit in the feedback loop.

The AGC circuit consists of an amplitude converter, a comparator, and a gaincontrolled amplifier, as shown in Fig. 3. The amplitude converter was made of the rmsto-dc converter AD736 (Analog Devices) with a divided-voltage input, which outputted an amplitude signal $V_{\text {a }}$ proportional to the rms of the input signal. The amplitude voltage was fed to the comparator made of the low-offset amplifier AD712 (Analog Devices). The comparator compared the vibration amplitude $V_{\text {a }}$ with the reference voltage $V_{\text {ref }}$ and controlled the gain of the gain-controlled amplifier. This comparator formed a Miller integrator of time constant $2 \pi R_{\mathrm{i}} C_{\mathrm{i}}$ to give a delayed response. A large feedback resistor $R_{\mathrm{f}}$ was added to prevent the saturation of the integrator. As the resonator device has a delay against amplitude control due to its $Q$ factor, the response has a time constant of approximately $Q / f$, where $f$ is the natural frequency of the resonator. Our device therefore had a $9.5 \mathrm{~ms}$ time constant. If the AGC responds much more rapidly than the device time constant, the loop gain instantly reaches the maximum or minimum before the device amplitude changes. Additionally, AD736 has a settling time ${ }^{(20)}$ estimated to be roughly $2 \mathrm{~ms}$ with the use of an average capacitance of $1 \mu \mathrm{F}$. We adjusted $R_{\mathrm{i}}, C_{\mathrm{i}}$, and $R_{\mathrm{f}}$ to make the time constant of the circuit similar to the device time constant and tuned them by observing the stability of vibration amplitude. We obtained good stability 


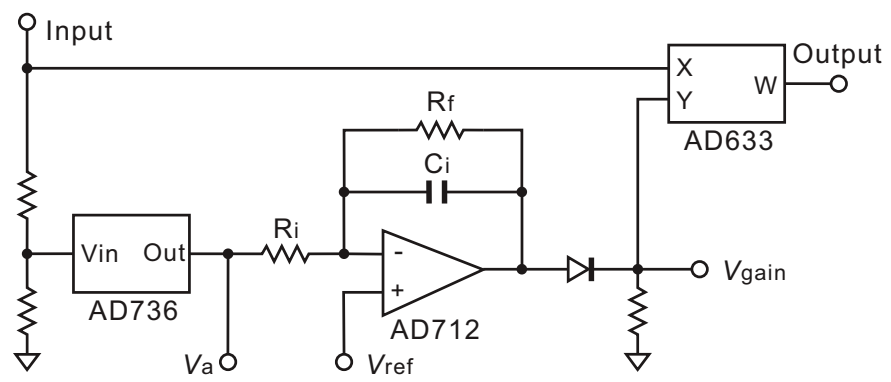

Fig. 3. Electric circuit for amplitude control of vibration.

against a wide range of amplitudes when we set $R_{\mathrm{i}}=4.5 \mathrm{k} \Omega, C_{\mathrm{i}}=0.22 \mu \mathrm{F}$, and $R_{\mathrm{f}}=1.2$ $\mathrm{M} \Omega$, where the time constant of the integrator was estimated to be $6.2 \mathrm{~ms}$. This value was in the same range as that in the case with an estimated device time constant of 9.5 ms. The adjustment of the circuit time constant was crucial in our system for stabilizing the vibration.

The output of the comparator was connected to the $\mathrm{Y}$ input of the analog multiplier AD633 (Analog Devices) in which the output $\mathrm{W}$ was controlled as $V_{\mathrm{W}}=V_{\mathrm{X}} V_{\mathrm{Y}} /(10 \mathrm{~V})$, where $V_{\mathrm{X}}, V_{\mathrm{Y}}$, and $V_{\mathrm{W}}$ were the voltages of the $\mathrm{X}, \mathrm{Y}$, and $\mathrm{W}$ terminals, respectively. ${ }^{(21)} \mathrm{We}$ also denote the voltage of the $\mathrm{Y}$ input as $V_{\text {gain }}$. This multiplier works as a gain-controlled amplifier when $V_{\text {gain }}$ is a dc-like signal. A diode was inserted to prevent negative values of the input $\mathrm{Y}$. As a result, the output $\mathrm{W}$ became the signal proportional to the input $\mathrm{X}$ with a controlled gain to maintain a constant amplitude according to the reference voltage. The loop gain of the circuit was adjusted to make $V_{\text {gain }}=5.0 \mathrm{~V}$ at the stable oscillation.

As the electrostatic sensing element in our resonator outputs the signal proportional to the rotational deflection, i.e., the bending angle of the resonator, the electric amplitude control in our test system means that the fatigue test is performed under constant deflection amplitude. It is impossible for our test device to measure or control the loading force because the device has no force-monitoring function. Force-controlled fatigue tests will become possible if stress-sensor-like piezoresistive elements are fabricated in the device properly.

\subsection{Frequency measurement}

Vibration frequency is one of the most important parameters that indicate the vibration conditions. Vibration frequency can be used to evaluate a test specimen, and to measure crack propagation through a change in compliance. ${ }^{(13)}$ A commercial frequency counter is one of the best choices for high-resolution frequency measurement. However, we employed the one-chip frequency-to-voltage converter ADVFC32 (Analog Devices) owing to its compatibility for a parallel-test system. This IC outputs a voltage proportional to the frequency that we denote $V_{\mathrm{f}}$. The frequency resolution was about 200 $\mathrm{Hz}$ in our system, which was sufficient for judging the device life, but was insufficient for observing a very small frequency shift due to crack propagation. 


\subsection{System fabrication}

We built twelve identical oscillation circuits in one system for parallel-test operation. Figure 4(a) shows the sample board in which twelve sets of a test device socket, a charge amplifier, and a high-voltage amplifier were installed. Four test devices were inserted on the board in this picture. This board can be set in a small environment-controlled chamber. Our method has the advantage of ease of precise environmental control over the use of large fatigue test machines. Actually, we could maintain the temperature and humidity of the test device at $23.0 \pm 0.1^{\circ} \mathrm{C}$ and $50 \pm 1 \% \mathrm{RH}$, respectively, for 7 days, using a small environment-controlled chamber. Temperature and humidity were recorded using a chart recorder during the test.

An oscillation circuit, except for that around the test device, was integrated in a subboard like that shown in Fig. 4(b) for each channel. Twelve sub-boards were gathered in a small case, as shown in Fig. 4(c), along with shared connectors and power supplies.

Each oscillation circuit had two voltage outputs (amplitude voltage $V_{\mathrm{a}}$ and frequency voltage $V_{\mathrm{f}}$ ) and one voltage input (reference voltage $V_{\text {ref }}$ ) per channel. We used an 8-channel analog output card and a 16-channel analog input card in one personal computer to perform the test. The numbers of I/O board channels allowed us to simultaneously perform eight parallel tests. It is easy to scale up the number of parallel tests if more parallel circuits and I/O ports can be added.

The waveform of the oscillation was observed and recorded using a storage oscilloscope (DL1620, Yokogawa Electric Corp.) with a large memory (8 MB). The recording of the whole waveform during the test was impossible because the number of test cycles usually exceeded $10^{6}$ and sometimes reached $10^{11}$. Therefore, we recorded the vibration waveform only at important moments such as fracture.
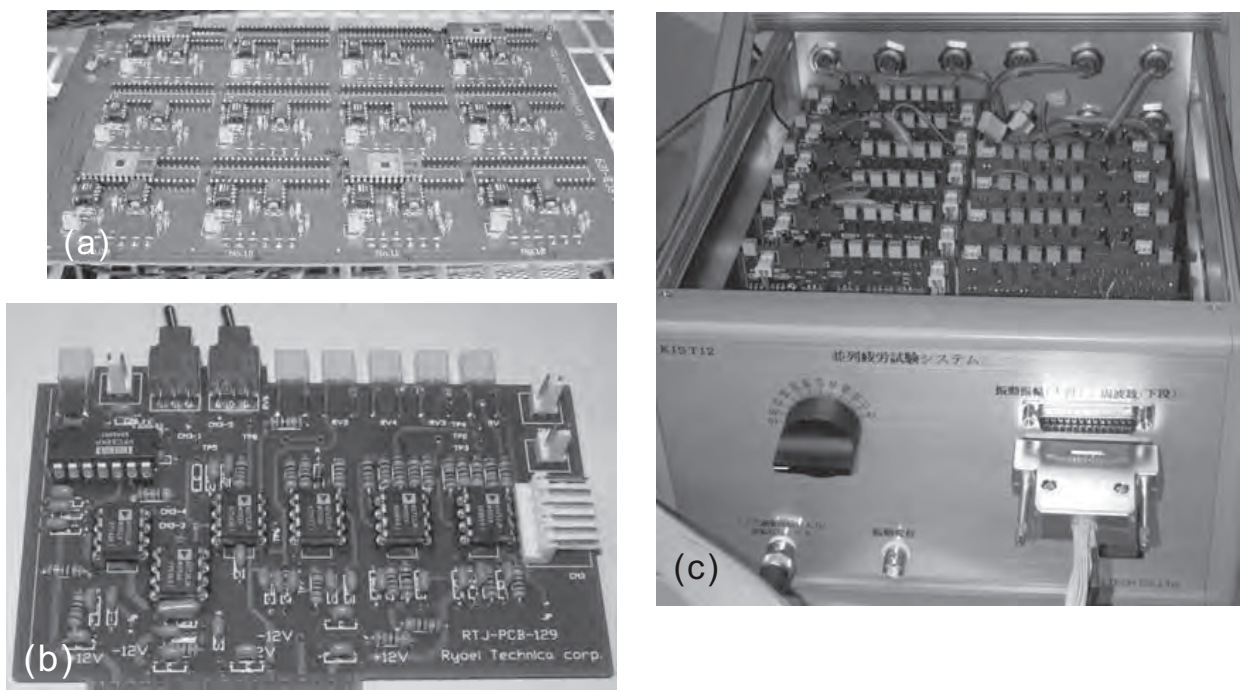

Fig. 4. Photographs of (a) sample board carrying four specimens, (b) vibration-control circuit for each channel, and (c) integrated twelve-channel test system. 
We developed a test control program using a compiler (Delphi) on the Windows XP operating system, as shown in Fig. 5. This program controls $V_{\text {ref }}$, acquires $V_{\mathrm{a}}$ and $V_{\mathrm{f}}$, displays these trends, records the data to the disk, and terminates the test at the preset duration. The power of all the systems except the environment-controlled chamber was maintained using an uninterruptible power supply.

\section{Measurements}

\subsection{Measurement procedures}

Before the fatigue test, we performed some preparatory tests on each test device. First, we confirmed the presence of the resonance at approximately $39 \mathrm{kHz}$ using external ac generator actuation that turns the feedback circuit off. If the resonator does not move due to fabrication errors or the shortening of electric circuit in the test chip, no resonance is observed in this test. A typical resonance curve has a bandwidth of 0.15 $\mathrm{kHz}$ corresponding to $\mathrm{Q}=370$, as shown in a previous study. ${ }^{(14)}$ Next, each device was examined to identify self-oscillation by switching the feedback circuit on with a small amplitude setting. Some devices were rejected for testing owing to unstable oscillation or lack of oscillation in this selection process.

After checking all the test devices separately, the fatigue test was started. At the beginning of the test, the amplitude setting $V_{\text {ref }}$ was linearly ramped to the preset voltage in $10 \mathrm{~s}$. We took this as the rise time because the test device showed instant

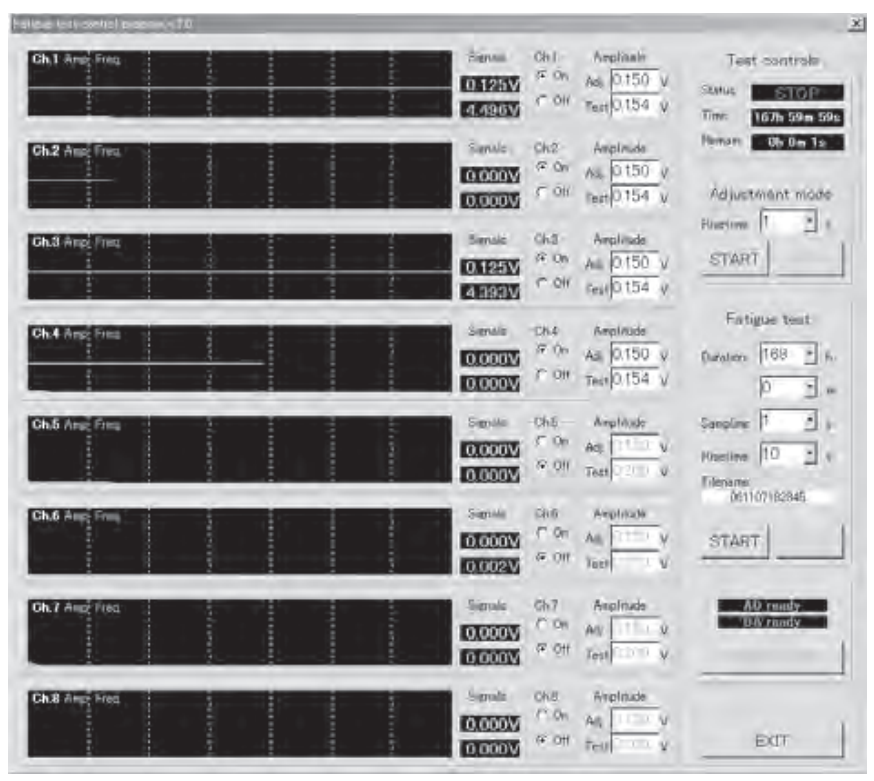

Fig. 5. Control screen of fatigue test on PC after four-channel parallel testing for $168 \mathrm{~h}$. 
failure owing to unstable oscillation when we changed the amplitude setting directly to the preset level, even if the time constant of the AGC was given properly. Since the oscillation settling time was on the order of milliseconds, a rise time of $1 \mathrm{~s}$ was sufficient to start oscillation without failure. However, a small amplitude fluctuation was observed for several seconds after reaching the preset amplitude. Therefore, we set the rise time to $10 \mathrm{~s}$.

We set the data acquisition interval to $1 \mathrm{~s}$ because a $\pm 5 \%$ accuracy in lifetime estimation can be expected even for a very short lifetime of $10 \mathrm{~s}$. A shorter interval is still possible; however, it results in a huge data size, which makes the analysis difficult. After starting the oscillation, the waveforms of all the channels were confirmed to be oscillating stably using the oscilloscope. Then, we waited for the test to finish, or for the test device to stop oscillating. The test pieces were examined under a microscope to determine whether it was broken or intact after terminating the fatigue test.

\subsection{Fatigue test results}

The data recorded in the control computer are shown in Fig. 6 for a test device that showed a very short lifetime. The time 0 is the start time of the fatigue test. The amplitude output $V_{\text {a }}$ gradually increased to the preset level in $10 \mathrm{~s}$ almost linearly except for $1 \mathrm{~s}$ at the beginning. The frequency output $V_{\mathrm{f}}$ exhibited almost constant values from the time of $2 \mathrm{~s}$. These data as well as results of the visual monitoring of $V_{\text {osc }}$ with an oscilloscope showed that the test device exhibited a stable and continuously amplitudecontrolled oscillation during the ramped rise procedure. The test device continued its stable oscillation after $10 \mathrm{~s}$, then suddenly stopped its oscillation at $27 \mathrm{~s}$. The two output

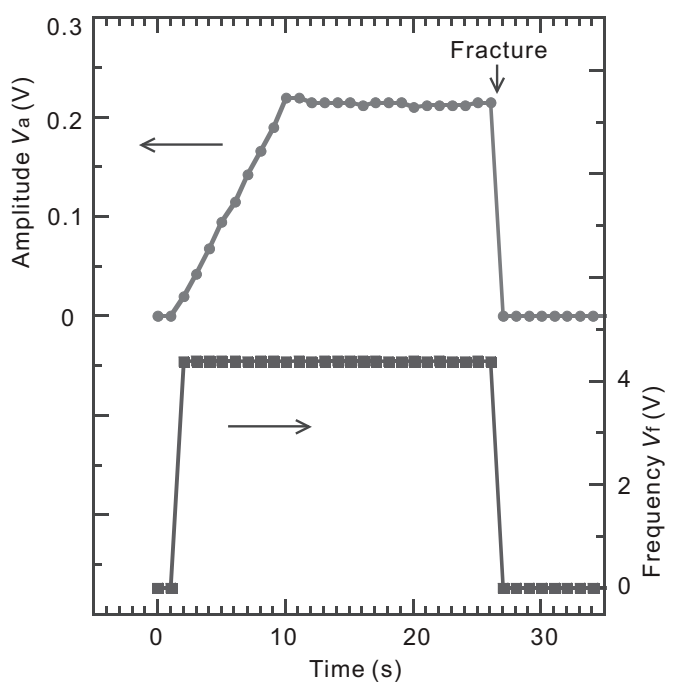

Fig. 6. Measured amplitude voltage $V_{\mathrm{a}}$ and frequency voltage $V_{\mathrm{f}}$ trends for short-lifetime specimen. 
voltages showed sudden drops to $0 \mathrm{~V}$, as shown in Fig. 6. This was the fracture of the test piece in the test device, and its lifetime was estimated to be $16.5 \pm 0.5 \mathrm{~s}$ from these data. The lifetime of $16.5 \mathrm{~s}$ was one of the shortest lifetimes under a relatively large loading in our test conditions. We observed a wide distribution of lifetimes from $16 \mathrm{~s}$ to more than $6.0 \times 10^{5} \mathrm{~s}$ ( 7 days) depending on the loading conditions. No frequency shifts have been observed in our measurement results within the low frequency resolution of about $200 \mathrm{~Hz}$. See previous reports for the experimental results. ${ }^{(14,19)}$

\subsection{Oscillation signals}

We also observed the oscillation signal $V_{\text {osc }}$, which was proportional to deflection angle, during the fatigue test. Figure 7 shows the $V_{\text {osc }}$ signal during the fatigue test for another short-lifetime ( $43 \mathrm{~s}$ ) test device approximately $15 \mathrm{~s}$ after the start of the test. The upper figure with a short time scale showed a sinusoidal oscillation signal at $39.14 \mathrm{kHz}$ with a constant amplitude. At the same time, the gain control voltage $V_{\text {gain }}$ exhibited a constant value of $5.0 \mathrm{~V}$. The lower figure showed a long-term stability of these signals. Only the envelope can be seen for $V_{\text {osc }}$. The oscillation was completely stable.

It was almost impossible to record all the oscillation signals during the test. However, we cannot predict the moment of fracture before it occurs. We obtained the oscillation signal at fracture employing the data storage and trigger functions of the oscilloscope. Several signals like $V_{\mathrm{a}}$ and $V_{\mathrm{f}}$ can be used as the trigger target. We finally chose the gain control voltage $V_{\text {gain }}$ for the trigger target because this signal was almost noise-free owing to the presence of an integration circuit working as a filter. Figure 8 shows the $V_{\text {osc }}$ and $V_{\text {gain }}$ signals at fracture. In the lower figure on a long time scale, the intensity of the gain control signal increased gradually after the oscillation stopped. When we set the
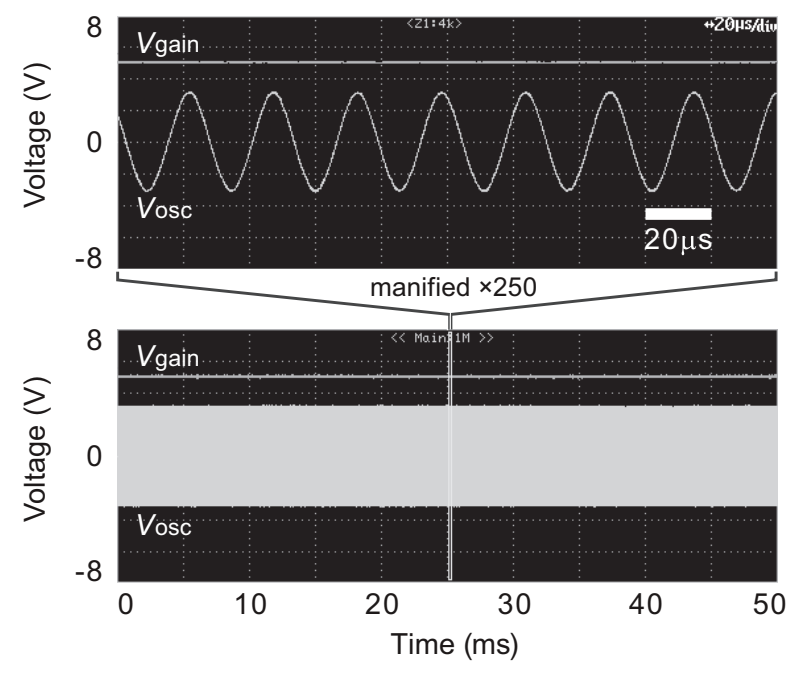

Fig. 7. Measured deflection signal $V_{\text {osc }}$ and gain voltage $V_{\text {gain }}$ at stable oscillation. The upper figure shows the magnified waveform on a time axis. 


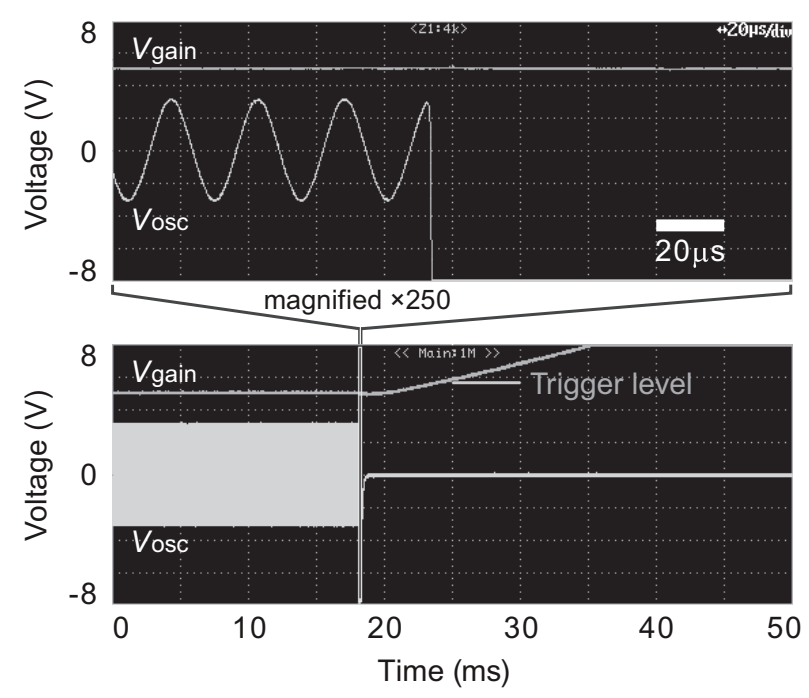

Fig. 8. Measured deflection signal $V_{\text {osc }}$ and gain voltage $V_{\text {gain }}$ at fracture. The upper figure shows the magnified waveform on a time axis.

trigger level to $5.6 \mathrm{~V}$ for the $V_{\text {gain }}$ signal of $5.0 \mathrm{~V}$ during stable oscillation, the recording was triggered $7 \mathrm{~ms}$ after fracture. It was short enough to record the moment of fracture because we used a $1 \mathrm{MB}$ memory and a $50 \mathrm{~ns}$ sampling time (full scale, $50 \mathrm{~ms}$ ). The upper figure shows the magnified signal at fracture. We could observe that the fracture occurred at the moment of almost maximum deflection and that the fracture was not induced by unstable oscillation or noise during the test.

\subsection{Parallel operation}

We attempted parallel testing with multiple test devices. However, we sometimes encountered a problem of unstable oscillation, which was seen only at parallel operations. Figure 9 shows $V_{\text {osc }}$ and $V_{\text {gain }}$ under unstable oscillation. The oscillation amplitude fluctuated about $\pm 20 \%$ with a period of $14 \mathrm{~ms}$ from the average amplitude. At the same time, gain voltage slightly fluctuated. The degree and period of fluctuation were dependent on tests. On the other hand, the test device showed a completely stable vibration in the case of single oscillation. We found that such an unstable oscillation occurred owing to interchannel interferences when multiple test devices have identical resonance frequencies. We have not specified yet where such interference takes place in our system. However, we could prevent this effect by selecting different resonance frequencies of the test devices before the test because the devices had small frequency differences among samples mainly owing to film thickness uniformity. We succeeded in suppressing the amplitude fluctuation within $\pm 4 \%$ although the maximum number of parallel tests was limited to within four. A perfect electric isolation is desired for largescale parallel testing. 


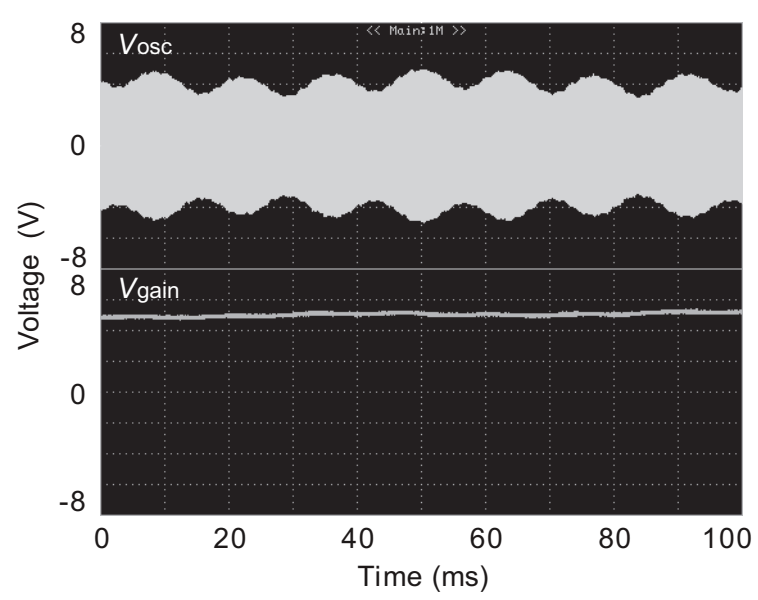

Fig. 9. Measured deflection signal $V_{\text {osc }}$ and gain voltage $V_{\text {gain }}$ for unstable oscillation due to interference effects.

\section{Conclusions}

The design, fabrication, measurement, and tuning procedures of a fatigue test system were described for amplitude-controlled parallel testing using self-oscillation. Amplitude-controlled oscillation was fundamental for performing the fatigue test reliably and the tuning of the time constant was crucial to obtaining stable oscillation considering the mechanical response of the test device. As the electric system was integrated on a small circuit board and had a simple interface with a control computer, it was easy to build a scaled-up, parallel-test system. Up to fourfold parallel testing was actually performed. A problem with the parallel testing system concerning signal interference was reported. Lifetime measurement and the acquisition of a deflection signal at fracture were successfully performed using the test system. We expect that such a test system will accelerate the reliability assessment of MEMS devices, as well as the characterization of microstructured materials for MEMS applications.

\section{Acknowledgments}

This research was supported by the New Energy and Industrial Technology Development Organization (NEDO) of Japan.

\section{References}

1 C. P. Chen and M. H. Leipold: J. Am. Ceram. Soc. 68 (1985) C-54.

2 C. P. Chen and M. H. Leipold: Crack Growth in Single Crystal Silicon, Fracture Mechanics of Ceramics Vol. 8, eds. R. C. Bradt et al. (Plenum Press, New York, 1986) p. 285. 
3 J. A. Connally and S. B. Brown: Science 256 (1992) 1537.

4 H. Kahn, R. Ballarini, R. L. Mullen and A. H. Heuer: Proc. R. Soc. Lond. A 455 (1999) 3807.

5 C. L. Muhlstein, S. B. Brown and R. O. Ritchie: Sens. Actuators, A 94 (2001) 177.

6 W. N. Sharpe, Jr. and J. Bagdahn: Mech. Mater. 36 (2004) 3.

7 T. Tsuchiya, A. Inoue, J. Sakata, M. Hashimoto, A. Yokoyama and M. Sugimoto: Tech. Dig. 16th Sensor Symp. (IEEJ, Kawasaki, 1998) p. 277.

8 T. Namazu and Y. Isono: J. Microelectromech. Systems 18 (2009) 129.

9 T. Ando, M. Shikida and K. Sato: Sens. Actuators, A 93 (2001) 70.

10 J. Bagdahn and W. N. Sharpe, Jr.: Sens. Actuators, A 103 (2003) 9.

11 Y. Yamaji, K. Sugano, O. Tabata and T. Tsuchiya: Tech. Dig. 20th MEMS Conf. (IEEE, Kobe, 2007) p. 267.

12 T. Kawai, S. Amaki, J. Gaspar, P. Ruther, O. Paul and S. Kamiya: Tech. Dig. 21st IEEE MEMS Conf. (IEEE, Tucson, 2008) p. 431.

13 O. N. Pierron and C. L. Muhlstein: J. Microelectromech. Systems 15 (2006) 111.

14 T. Ikehara and T. Tsuchiya: J. Micromech. Microeng. 18 (2008) 075004.

15 C. L. Muhlstein, E. A. Stach and R. O. Ritchie: Acta Materialia 50 (2002) 3579.

16 H. Kahn, L. Chen, R. Ballarini and A. H. Heuer: Acta Materialia 54 (2006) 667.

17 S. Bhowmick, J. J. Meléndez-Martínez and B. R. Lawn: Appl. Phys. Lett. 91 (2007) 201902.

18 D. H. Alsem, R. Timmerman, B. L. Boyce, E. A. Stach, J. Th. M. De Hosson and R. O. Ritchie: J. Appl. Phys. 101 (2007) 013515.

19 T. Ikehara and T. Tsuchiya: Tech. Dig. 21st IEEE MEMS Conf. (IEEE, Tucson, 2008) p. 435.

20 Data Sheet of Low Cost, Low Power, True RMS-to-DC Converter, AD736, Rev. G (Analog Devices Inc., 2006).

21 Data Sheet of Low Cost, Analog Multiplier, AD633, Rev. E (Analog Devices Inc., 2002). 